\title{
Do Triptan Antimigraine Medications Interact with SSRI/SNRI Antidepressants? What Does Your Decision Support System Say?
}

\author{
Stephen J. Kogut, MBA, PhD
}

$\mathrm{D}$ rug risks may be ignored or underappreciated, overemphasized, misinterpreted, or in some cases presented in ways that do not wholly reflect the evidence base and clinical experience. Often the available evidence pertaining to drug interactions is grounded upon theoretical concerns, very small trials, or case reports, and thus it is often very challenging to balance the potential risks and benefits of a particular therapy when drug interactions are present. While prescribers and pharmacists may fail to heed interaction alerts provided by decision support systems, it is also possible that the overstatement of risks can cause providers to avoid utilizing a clinically beneficial therapy. The case of the interaction between triptan antimigraine medications and selective serotonin reuptake inhibitor/selective serotonin-norepinephrine reuptake inhibitor (SSRI/SNRI) antidepressants provides an example of how the presentation of drug risk may unduly influence the utilization of drug therapy.

\section{The Risk of Serotonin Syndrome Associated with Concomitant Use of Triptans and SSRIs/SNRIs}

The U.S. Food and Drug Administration (FDA), in its endeavors to govern the balance between drug benefits and safety concerns, faces the tremendous challenge of evaluating emerging evidence and guiding the assimilation of new knowledge into prescribing recommendations. This paper presents one such example, as in 2006, the FDA issued an alert to health care professionals regarding the potential risks associated with the combined use of 5-hydroxytryptamine receptor agonist (i.e., triptan) antimigraine medications and certain antidepressant medications, specifically SSRIs and SNRIs. ${ }^{1}$ This advisory, which remains posted at the FDA.gov website as of August 11, 2011, includes a headline that warns health care providers and the general public that the combination of these medications may result in "Potentially Life-threatening Serotonin Syndrome." The advisory recommends that physicians should evaluate the risk of serotonin syndrome against the expected benefit of using this drug combination and discuss the possibility of serotonin syndrome with their patients utilizing this combination. The FDA also recommends that prescribers follow patients closely for the development of signs of serotonin syndrome, which are noted to include autonomic instability, respiratory failure, and coma. ${ }^{1}$

The FDA issued this alert based upon its review of 27 case reports describing cases of serotonin syndrome occurring within the context of concomitant use of triptan antimigraine agents and SSRI or SNRI antidepressants. According to the alert, 2 of these 27 patients experienced life-threatening symptoms, and approximately one-half of the 27 patients required hospitalization. No fatalities were reported. The FDA stated that "it is expected that concomitant use of SSRIs or SNRIs and triptans would result in higher serotonin levels than the serotonin levels observed with the use of SSRIs, SNRIs, or triptans alone, potentially leading to serotonin syndrome." ${ }^{1}$

The FDA qualified its alert as reflecting its initial review of these cases and stated that a conclusion has not yet been reached. ${ }^{1}$ However, a related public health advisory issued on July 19, 2006, stated that "The FDA has determined that serotonin syndrome occurs with combined use of triptans and a SSRI or SNRI through reports describing serotonin syndrome in people taking these medications together." ${ }^{2}$ The product labeling for triptan antimigraine medications warns of the risk of serotonin syndrome as precipitated by concomitant use with SSRI or SNRI antidepressants. For example, the product labeling for sumatriptan states that serotonin syndrome may occur when the medication is co-prescribed with SSRI/SNRI antidepressants, necessitating close monitoring, particularly at the onset of treatment and with dose escalations. ${ }^{3}$

\section{Should Case Reports Serve as the Basis for Causal Inference?}

Some neuropsychiatric experts have questioned the FDA case reports and the biologic plausibility underlying the purported interaction between triptan medications and antidepressants. Their skepticism is well summarized by Gillman (2010) in a review article published in the journal Headache: "there is neither significant clinical evidence, nor theoretical reason, to entertain speculation about serious [serotonin syndrome] from triptans and SSRIs." ${ }^{4}$ Others have opined that the FDA may have been unnecessarily overcautious in its warning, pointing to the fact that the evidence currently available to support this drug interaction is derived primarily from case reports, which provide a low degree of scientific validity, and questioning the science underlying the theory that a pharmacologic interaction exists that would increase the risk of serotonin syndrome..$^{5-7}$ Yet, because serotonin syndrome is indeed a serious, lifethreatening condition, the FDA's decision to alert prescribers of this possible risk is understandable, even though a cause-andeffect relationship is unclear. 
To further evaluate the accuracy of the case reports, Evans analyzed the cases included in the FDA review, ${ }^{8}$ and also engaged in a broader effort involving other experts from the field of headache management, to review the FDA cases in addition to other reports of serotonin syndrome associated with the use of these medications appearing in the published literature. ${ }^{7}$ Diagnostic criteria were applied to determine whether the described cases of serotonin syndrome could be clinically validated. When applying the Sternback Criteria for diagnosis of serotonin syndrome, 10 of the 29 cases reviewed by the FDA were validated; when applying the more robust Hunter Criteria, no cases were validated. ${ }^{7}$ Among the cases confirmed using the Sternback criteria, the reviewers noted that information necessary to rule out other conditions was unavailable, thus calling into question the accuracy of diagnosis using this method. The reviewers concluded that the coprescribing of triptan medications with SSRI/SNRI antidepressants should not be prohibited based on these cases. Yet, they also recognized that the data do not exclude the possibility of an increased risk of serotonin syndrome, noting that "Triptans when administered with SSRIs or SNRIs might rarely precipitate serotonin syndrome". ${ }^{8}$

In contrast to the FDA's mention of the biological plausibility of the interaction ${ }^{1}$ Evans et al. (2010) described the risk of serotonin syndrome occurring from the co-prescribing of triptans and SSRI/SNRI antidepressants as seeming to be "implausible." Triptan medications demonstrate weak affinity for 5-HT(1A) and no activity at 5-HT2 receptors, ${ }^{3}$ which are believed to be mechanistically implicated in the development of serotonin syndrome. ${ }^{9}$ Evans et al. suggested that because of the life-threatening nature of serotonin syndrome, prescribers should remain attentive to the possibility of the development of serotonin syndrome, ${ }^{7}$ a position that aligns in some measure with the FDA's alert. However, it was the viewpoint of Evans et al. that the increased risk of serotonin syndrome associated with the concomitant use of triptans and SSRI or SNRI medications is not proven, and the FDA should reassess the available evidence to determine if its alert and the warning statement appearing in the triptan labeling should be revised or removed. These views are presented in an American Headache Society (AHS) Position Paper published in 2010, which declared that "inadequate data are available to determine the risk of serotonin syndrome with the addition of a triptan to SSRIs/SNRIs or with triptan monotherapy" ${ }^{7}$ The AHS Position Paper also stated that "The currently available evidence does not support limiting the use of triptans with SSRIs or SNRIs, or the use of triptan monotherapy, due to concerns for serotonin syndrome." ${ }^{\top}$

\section{Epidemiologic Evidence Suggests Very Low or No Risk}

Practitioners often encounter depressed patients seeking treatment for headache, as migraine and other types of severe headache are a common malady affecting the U.S. adult population. When not contraindicated, triptan medications are a mainstay of treatment for a migraine of moderate to severe intensity. ${ }^{10}$ In an analysis performed using data from the 1999-2004 National Health Examination and Nutrition Survey (NHANES), Kalaydjian and Merikangas (2008) found that 28\% of adult females and 15\% of adult males reported experiencing at least 1 episode of severe headache or migraine during the past 3 months. ${ }^{11}$ They also observed that sufferers of migraine or other types of severe headache were nearly 3 times as likely to report having major depression or panic disorder. Other survey data suggest a strong association between migraine and a range of psychiatric disorders, including depression, bipolar disorder, and social phobia. ${ }^{12}$

The epidemiologic data suggest that it is not uncommon for practitioners to encounter clinical scenarios where the prescribing of both triptan medications and antidepressants may be considered. Results from 2 analyses reveal that triptans and SSRI or SNRI medications were co-prescribed with high frequency prior to the FDA advisories of 2006. Tepper et al. (2003) examined claims from a large pharmacy benefit management (PBM) company and found that approximately 1 of 5 patients prescribed a triptan medication also received prescriptions for SSRI antidepressants..$^{13}$ Sclar et al. (2008), analyzing data from the 2003-2004 National Ambulatory Medical Care Survey (NAMCS), estimated that an average of approximately 700,000 U.S. outpatient visits per year included mention of the prescribing of both a triptan medication and an SSRI or SNRI antidepressant. ${ }^{14}$ While Sclar et al. characterized the drug combination as "potentially fatal," their finding that nearly 700,000 Americans are prescribed the combination yearly, taken in contrast with the very small numbers of questionable case reports received by the FDA and/or reported in the literature, suggests that the increased risk of serotonin syndrome is extremely low or perhaps nonexistent. Wenzel et al. (2008) more strongly opined that "years of clinical experience show that this combination of medications has been taken by millions of patients without any known serotonin syndrome consequences". ${ }^{6}$

If an increased risk does exist, it is not possible to quantify the magnitude of risk from the case reports cited by the FDA in the absence of a denominator of all users of this combination of medications during the time frame, and recognizing that not all cases would be reported through the FDA MedWatch program. Moreover, if this risk exists, it is not clear whether it is any greater or less than the risk of severe hypersensitivity to triptan products themselves, which is borne by prescribers and patients with each incident prescribing.

The epidemiologic research of Tepper et al. and Sclar et al. describe the co-prescribing of these medications during periods that preceded the FDA alert of 2006. ${ }^{13,14}$ There appears to be no similar published epidemiologic research describing the co-prescribing of these medications during the years 
following the FDA alert. A replication of the Sclar et al. analysis in particular, utilizing more recent data from the NAMCS, would provide insight regarding the impact of the FDA's advisory on the co-prescribing of these medications. However, pharmacoepidemiologic studies assessing the outcomes of patients utilizing this combination of medications are of higher priority.

\section{Warning! Presentation of the Interaction in Leading Clinical Decision Support Applications}

The FDA alert and the precautions regarding serotonin syndrome occurring as a result of interaction with SSRI/SNRI antidepressant medications detailed in the prescribing information for triptan medications have been incorporated into leading stand-alone drug knowledge databases and integrated within clinical decision support systems. For example, the online drug interaction tools offered by Drugs. $\mathrm{Com}^{15}$ (Cerner Multum, Inc., Denver, CO) presently identifies the interaction between sumatriptan and fluoxetine as "major", while the interaction is also described as being in the category of "major" by Micromedex 2.0 (Thomson Reuters, New York, NY) ${ }_{16}^{16}$ ClinicalPharmacology (Elsevier/Gold Standard, Tampa, FL), ${ }^{17}$ and LexiComp Online (Lexi-Comp, Inc., Hudson, OH) ${ }^{18}$ The cautions provided by these applications are strongly worded and imply significant risk of serious patient harm. The Micromedex 2.0 product states that the "coadministration of a triptan, such as sumatriptan, and an SSRI, such as fluoxetine, may result in a life-threatening condition called serotonin syndrome," while LexiComp includes the headline "Consider therapy modification" following its "Risk Rating" heading.

The monograph for sumatriptan within the Micromedex 2.0 product presents a section of "precautions" for the interaction between sumatriptan and SSRI/SNRI antidepressants, which includes the statement that "serotonin syndrome (e.g., mental status changes, autonomic instability, neuromuscular aberrations, and/or gastrointestinal symptoms) may occur, particularly during concomitant use of SSRIs or serotonin norepinephrine reuptake inhibitors."16 The incidence of serotonin syndrome resulting from the interaction is not reported, nor is it noted as unknown or extremely rare. The evidence cited by Micromedex 2.0 to substantiate this precaution is the prescribing information for various sumatriptan formulations. Neither the Micromedex 2.0 database nor the cited prescribing information rates the strength of evidence supporting the warning, although some additional detail regarding the nature of evidence being from case reports is provided in a different section of the monograph. None of the aforementioned drug information resources mentions the American Headache Society (AHS) Position Paper, which declares that the currently available data are inadequate for concluding that an increased risk of serotonin syndrome exists or for serving as the basis for avoiding the use of triptan medications in patients utilizing
SSRI or SNRI antidepressants. ${ }^{7}$

This author suggests that clinical decision support systems should offer more than reformatting and streamlining of the drug product label and existing FDA advisories. Evidencebased decision making requires the assimilation of all pertinent information, such as position papers from prominent medical specialties. Clinical decision support applications that include knowledge from all available sources will yield greater utility for achieving better and safer patient outcomes.

\section{Improving the Ability to Manage Drug-Drug Interactions}

It is not the intent of this commentary to provide a definitive statement on whether triptan drugs interact with SSRI/SNRIs to cause serotonin syndrome. It has been approximately 5 years since the FDA published its alerts regarding the risk of serotonin syndrome when combining these medications and, as discussed above, several experts have published their opinion that the FDA has overstated the nature of this risk. The issue is illustrative in further considering how our health care system's technologies present risk information, and how practitioners incorporate this information in the treatment and monitoring of their patients.

Malone et al. (2005) found that a range of clinically important drug-drug interactions occur with high frequency in the U.S. health care system. ${ }^{19}$ Their results suggest, at least in part, either that prescribers are unaware of these drug interactions or that they accept the risk of harm associated with drug interactions, presumably believing that the benefits outweigh the risk and that the patient can be effectively monitored. This premise necessitates that pharmacists and prescribers have an accurate understanding of the nature of the risks. One may wonder if prescribers' perceptions of drug interaction risks have been shaped by being too frequently alerted to theoretical risks that have not occurred in their practices and thus are deemed to be either unfounded or extremely unlikely.

Ideal drug interaction alerts should note the nature of evidence establishing the interaction, the magnitude of risk and its consequences, and how the interaction juxtaposes with the patient's personal characteristics and clinical circumstance. In this author's opinion, the case of the purported interaction between SSRI/SNRI antidepressants and triptan antimigraine medications is an example of the failure of decision support systems to accurately portray risk information to practitioners. The pharmacoepidemiologic literature includes countless descriptions of controversies and suspicions of possible risks associated with specific drugs. Those compiling data within drug knowledge databases must consider the strength and breadth of evidence and generalizability of the evidence when translating information into decision support systems.

Decision support systems have a fundamental role in supporting drug utilization review functions, including drugdrug interaction screening. However, it has been more than 
a decade since the Institute of Medicine's Crossing the Quality Chasm report stated, in regards to clinical decision support, that "changes under way in health care and computing will likely result in the development of far superior tools in the near future..."20 Clinical decision support systems and their alerts are poorly effective in the management of drug-drug interactions, as evidenced by their lack of standardization and inability to detect significant interactions ${ }^{21}$ their insufficient detail and lack of suggestions for patient management, ${ }^{22}$ and their failure to provide appropriate clinical justification..$^{23,24}$ Notwithstanding the advances in system adoptions and integration of these systems into practice, the touted value of clinical decision support in promoting quality in medication use has yet to be fully realized, particularly with regard to managing drug-drug interactions and as noted by the degree to which alerts are ignored. The example of the purported interaction between triptan antimigraine drugs and SSRI/SNRI antidepressants presents a case where risk may be overstated, potentially resulting in a patient's failing to receive a beneficial therapy. Whether considering important risks that are underappreciated or lesser risks that are overstated, these scenarios can be addressed by improving how evidence is synthesized, interpreted and presented to decision makers.

A multistakeholder conference convened in October 2009 to discuss critical issues associated with drug-drug interactions identified the following as highest priorities for further study and action: population-based studies of drug-drug interactions, explicit evaluation of the quality and adequacy of current evidence, and improved integration of evidence in knowledge databases and clinical decision support systems. ${ }^{25}$ The first 2 priorities identified by this group pertain to our health care system's inability to adequately weigh the benefits against the risks of interacting medications when used across varied clinical circumstances. The lack of data describing drug therapy outcomes that can effectively inform treatment decisions has led to our nation's commitment of more than $\$ 1$ billion towards comparative effectiveness research via the American Recovery and Reinvestment Act of 2009. ${ }^{26}$ It appears from a review of the listings of funded projects provided by the U.S. Government Accountability Office that apparently no resources have been directed towards projects specifically evaluating the consequences of particular drug-drug interactions. ${ }^{27}$

Meanwhile, the Health Information Technology for Economic and Clinical Health (HITECH) Act has directed approximately $\$ 20$ billion towards improvements in health information technologies capable of alerting providers to drug-drug interactions. ${ }^{28}$ This is occurring while scant public resources are being directed towards improving the evidence base and clinical decision support systems from which these drug interaction alerts are generated. The presumed clinical and economic benefits to be reaped from our nation's investment in health information technologies depends upon the incorporation of valid, pertinent, and actionable information to support pharmacologic decision making. The current inadequacy of the existing evidence base detailing medication risks generally and drug interactions specifically must gain greater awareness among health system stakeholders.

It will be interesting to observe how prescribers' assessment of interaction risks and their co-prescribing of interacting drugs may change with the HITECH Act's requirement for meaningful use of electronic health records, which includes a criterion that places computerized drug interaction screening more squarely within the prescriber's domain. The clear presumption behind the meaningful use requirement that positions drug interaction screening upstream in the prescribing process is that electronic decision support systems contain valid and actionable information that informs the risk-tobenefit ratio in deciding whether a medication can be utilized with an acceptable margin of safety.

As Dr. Avedis Donabedian, a renowned physician and health care quality improvement pioneer, asserted: "The degree of quality is, therefore, the extent to which the care provided is expected to achieve the most favorable balance of risks and benefits." ${ }^{29}$ When considering drug-drug interactions, determining that balance requires improvements in the existing evidence base and the utility of decision support systems, and in how risk information is presented, interpreted, and applied.

\section{Author}

STEPHEN J. KOGUT, MBA, PhD, is Associate Professor, Department of Pharmacy Practice, University of Rhode Island, Kingston, Rhode Island.

AUTHOR CORRESPONDENCE: Stephen J. Kogut, MBA, PhD, Pharmacy Practice, University of Rhode Island, 41 Lower College Rd., Kingston, RI 02881. Tel.: 401.874.5370;

E-mail:kogut@URI.edu.

\section{DISCLOSURES}

The author reports no financial or other conflicts of interest related to the subjects or products discussed in this article.

\section{REFERENCES}

1. U.S. Food and Drug Administration. Information for healthcare professionals: selective serotonin reuptake inhibitors (SSRIs), selective serotonin-norepinephrine reuptake inhibitors (SNRIs), 5-hydroxytryptamine receptor agonists (triptans). FDA ALERT [7/2006]: Potentially life-threatening serotonin syndrome with combined use of SSRIs or SNRIs and triptan medications. July 2006, updated January 21, 2010. Available at: http://www.fda.gov/Drugs/ DrugSafety/PostmarketDrugSafetyInformationforPatientsandProviders/ DrugSafetyInformationforHeathcareProfessionals/ucm085845.htm. Accessed August 12, 2011. 
2. U.S. Food and Drug Administration. Public health advisory-combined use of 5-hydroxytryptamine receptor agonists (triptans), selective serotonin reuptake inhibitors (SSRIs) or selective serotonin/norepinephrine reuptake inhibitors (SNRIs) may result in life-threatening serotonin syndrome. July 19, 2006, updated June 22, 2010. Available at: http://www.fda.gov/Drugs/ DrugSafety/PostmarketDrugSafetyInformationforPatientsandProviders/ DrugSafetyInformationforHeathcareProfessionals/PublicHealthAdvisories/ ucm124349.htm. Accessed August 12, 2011.

3. Imitrex (sumatriptan) Tablets. GlaxoSmithKline. February 2010. Available at: http://us.gsk.com/products/assets/us_imitrex_tablets.pdf. Accessed August 12, 2011.

4. Gillman PK. Triptans, serotonin agonists, and serotonin syndrome (serotonin toxicity): a review. Headache. 2010;50(2):264-72.

5. Schuman ES. Is an FDA alert harming patients? JAAPA. 2009;22(5):55. Available at: http://www.jaapa.com/is-an-fda-alert-harming-patients/article/136691/. Accessed August 12, 2011.

6. Wenzel RG, Tepper S, Korab WE, Freitag F. Serotonin syndrome risks when combining SSRI/SNRI drugs and triptans: is the FDA's alert warranted? Ann Pharmacother. 2008;42(11):1692-96.

7. Evans RW, Tepper SJ, Shapiro RE, Sun-Edelstein C, Tietjen GE. The FDA alert on serotonin syndrome with use of triptans combined with selective serotonin reuptake inhibitors or selective serotonin-norepinephrine reuptake inhibitors: American Headache Society position paper. Headache. 2010;50(6):1089-99.

8. Evans RW. The FDA alert on serotonin syndrome with combined use of SSRIs or SNRIs and Triptans: an analysis of the 29 case reports. MedGenMed. 2007;9(3):48. Available at: http://www.ncbi.nlm.nih.gov/pmc/ articles/PMC2100123/. Accessed August 12, 2011.

9. Ener RA, Meglathery SB, Van Decker WA, Gallagher RM. Serotonin syndrome and other serotonergic disorders. Pain Med. 2003;4(1):63-74.

10. Silberstein SD. Practice parameter: evidence-based guidelines for migraine headache (an evidence-based review): report of the Quality Standards Subcommittee of the American Academy of Neurology.

Neurology. 2000;55(6):754-62. Available at: http://www.neurology.org/content/55/6/754.long. Accessed August 12, 2011.

11. Kalaydjian A, Merikangas K. Physical and mental comorbidity of headache in a nationally representative sample of US adults. Psychosom Med. 2008;70(7):773-80. Available at: http://www.ncbi.nlm.nih.gov/pmc/articles/ PMC2933379/. Accessed August 12, 2011.

12. Jette N, Patten S, Williams J, Becker W, Wiebe S. Comorbidity of migraine and psychiatric disorders-a national population-based study. Headache. 2008;48(4):501-16.

13. Tepper S, Allen C, Sanders D, Greene A, Boccuzzi S. Coprescription of triptans with potentially interacting medications: a cohort study involving 240,268 patients. Headache. 2003;43(1):44-48.

14. Sclar DA, Robison LM, Skaer TL. Concomitant triptan and SSRI or SNRI use: a risk for serotonin syndrome. Headache. 2008;48(1):126-29.

15. Drugs.com. Drug Interactions Checker. Available at: http://www.drugs. com/drug_interactions.html. Accessed August 12, 2011.
16. Thomson Reuters. Micromedex 2.0. Product Monograph: Sumatriptan. Accessed June 22, 2011 via license through University of Rhode Island College of Pharmacy. Product description available at: http://www.micromedex.com/. Accessed August 12, 2011.

17. Elsevier/Gold Standard. Clinical Pharmacology Drug Interaction Report: Sumatriptan. Accessed June 22, 2011 via license through University of Rhode Island College of Pharmacy. Product description available at: http:// www.clinicalpharmacology.com. Accessed August 16, 2011.

18. Lexicomp Online Interaction Monograph: Sumatriptan + Fluoxetine. Accessed June 22, 2011 via license through University of Rhode Island College of Pharmacy. Product description available at: http://www.lexi.com/ institutions/universities.jsp?id=academic. Accessed August 16, 2011.

19. Malone DC, Hutchins DS, Haupert H, et al. Assessment of potential drug-drug interactions with a prescription claims database. Am J Health Syst Pharm. 2005;62(19):1983-91.

20. Committee on Quality of Health Care in America (IOM). Using Computer-Based Clinical Decision Support Systems. In: Crossing the Quality Chasm: A New Health System for the 21st Century. Washington DC: National Academy Press; 2001:154.

21. Saverno KR, Hines LE, Warholak TL, et al. Ability of pharmacy clinical decision-support software to alert users about clinically important drugdrug interactions. J Am Med Inform Assoc. 2011;18(1):32-37.

22. Ko Y, Abarca J, Malone DC, et al. Practitioners' views on computerized drug-drug interaction alerts in the VA system. J Am Med Inform Assoc. 2007;14(1):56-64.

23. Grizzle AJ, Mahmood MH, Ko Y, et al. Reasons provided by prescribers when overriding drug-drug interaction alerts. Am J Manag Care. 2007;13(10):573-78.

24. Isaac T, Weissman JS, Davis RB, et al. Overrides of medication alerts in ambulatory care. Arch Intern Med. 2009;169(3):305-11. Available at: http:// archinte.ama-assn.org/cgi/content/full/169/3/305. Accessed June 27, 2011.

25. Hines LE, Murphy JE, Grizzle AJ, Malone DC. Critical issues associated with drug-drug interactions: highlights of a multistakeholder conference. Am J Health Syst Pharm. 2011;68(10):941-46.

26. Sullivan P, Goldmann D. The promise of comparative effectiveness research. JAMA. 2011;305(4):400-01.

27. U.S. Government Accountability Office. HHS research awards: use of recovery act and patient protection and affordable care act funds for comparative effectiveness research. June 14, 2011. Available at: http://www.gao. gov/new.items/d11712r.pdf. Accessed August 12, 2011.

28. Stark P. Congressional intent for the HITECH Act. Am J Manag Care. 2010;16(12 Suppl HIT):SP24-S28. Available at: http://www.ajmc.com/ publications/supplement/2010/AJMC_10dec_HIT/AJMC_10decHIT_Stark_ SP24tp28/. Accessed August 12, 2011.

29. Donabedian A. The definition of quality and approaches to its assesessment. In: Explorations in Quality Assessment and Monitoring: Volume 1. Ann Arbor, MI: Health Administration Press; 1980. 